NOTA TÉCNICA

\title{
EVALUACIÓN DE LEGUMINOSAS DE LOS GÉNEROS Vicia, LotusY Trifolium EN LA ZONA ALTA DE LA PROVINCIA DE HEREDIA, COSTA RICA
}

\author{
María Mesén ${ }^{1}$, William Sánchez ${ }^{1}$
}

\begin{abstract}
RESUMEN
La presente investigación se realizó en el año 1996, en una finca ubicada en el caserío Los Cartagos, distrito Santo Domingo, cantón Santa Bárbara, provincia de Heredia. La topografía de la finca es irregular y se encuentra a una la altitud de $2.050 \mathrm{msnm}$. La temperatura y precipitación promedio anual son de $16,0^{\circ} \mathrm{C}$ y $3.143 \mathrm{~mm}$, respectivamente. El objetivo de la investigación fue evaluar la adaptabilidad de una colección de 15 cultivares de leguminosas de los géneros Vicia, Lotus y Trifolium. Se evaluaron especies anuales, perennes y semiperennes, con las especies anuales se realizó únicamente una evaluación al inicio de la floración de las mismas y se utilizaron medidas de tendencia central. Con los demás cultivares se utilizó un diseño experimental de bloques completos al azar con arreglo de parcelas divididas. Los cultivares anuales presentaron altos rendimientos de materia seca, contenido de proteína cruda y digestibilidad in vitro de la materia seca (D.I.V.M.S). El forraje de mayor producción de biomasa fue la Vicia purpurea, Púrpura, con 6,3 t/ha de materia seca y el de menor producción fue el Trifolium alexandrinum, Nico, con 2,7 t/ha. Los cultivares perennes y semiperennes recibieron cortes cada seis semanas, durante dos años, al último corte sobrevivieron cinco cultivares, el Lotus corniculatos, Makú, los Trifolium pratense, Renegade y Quiñequeli y los Trifolium repens Arán e Italia con rendimiento promedio de materia seca de 1,1, 1,8,1,4, 0,8 y 1,2 t/ha/corte, respectivamente. Los valores de proteína cruda fueron muy similares, oscilaron entre 23,9 y $26,1 \%$. Se concluye que todos los cultivares anuales se adaptan a la zona. De los cultivares perennes o semiperennes sobrevivieron únicamente cinco, con los cuales se debe continuar la investigación bajo pastoreo y/o en asociación con gramíneas.
\end{abstract}

Palabras clave: Leguminosas, forrajes, valor nutritivo, producción de leche.

\section{INTRODUCCIÓN}

El sector lácteo ha cumplido con un papel de impacto en la evolución económica y social de los países. Costa Rica cuenta con la planta de mayor volumen de industrialización de leche en América Central y el Caribe, procesa aproximadamente $927.000 \mathrm{~kg}$ de leche diarios. Sin embargo, la tendencia mundial de libre comercio requiere de mayores niveles de eficiencia en los sistemas de producción, de manera tal que se pueda competir en la exportación a otras naciones (Villegas 2004).

En la mayoría de las empresas ganaderas el forraje sigue siendo la fuente alimenticia más común y de menor costo, el cual normalmente no suple los requerimientos de los animales de mediana y alta producción, debido a la limitada cantidad y calidad del forraje disponible.

1 Instituto Nacional de Innovación y Transferencia en Tecnología Agropecuaria (INTA). Costa Rica. 
En estos casos en la mayoría de las fincas las necesidades nutricionales se satisfacen mediante el uso de alimentos balanceados, basados en materias primas importadas, lo cual encarece los costos de producción en el rubro de alimentación, representando éstos hasta un $72,0 \%$ de los costos totales de alimentación en ganadería de leche especializada (MAG-IICA 1991).

En este sentido, las leguminosas en monocultivo o en asociación con gramíneas han sido reconocidas como fuentes de excelente forraje y como mejoradoras de la fertilidad del suelo. Su habilidad para fijar nitrógeno del aire y su alto contenido de proteína y minerales, las hacen indispensables en la alimentación animal (Bernal 1991).

Por lo anteriormente mencionado, se utilizó semilla sexual de una colección de leguminosas forrajeras para clima frío, con el objetivo de buscar variedades de leguminosas que se adapten a la zona alta lechera de Costa Rica.

\section{MATERIALES Y MÉTODOS}

El presente trabajo se desarrolló en el año 1996, en una finca ubicada en el caserío Los Cartagos, distrito Santo Domingo, cantón Santa Bárbara, provincia Heredia. La topografía de la finca es irregular y está a una la altitud de $2.050 \mathrm{msnm}$. La temperatura y precipitación promedio anual son de $16{ }^{\circ} \mathrm{C} y$ $3.143 \mathrm{~mm}$, respectivamente.

El terreno utilizado había sido cultivado anteriormente con el forraje Pennisetum clandestinum. Geomorfológicamente el suelo es de origen volcánico y taxonómicamente corresponde al orden de los andisoles (Bertsch et al. 1993).
El análisis de suelo (Cuadro 1) muestra que todos los elementos con excepción del $\mathrm{Fe}$, se encuentran dentro del rango óptimo, también presenta un valor adecuado en cuanto al porcentaje de acidez. Las relaciones $\mathrm{Ca} / \mathrm{Mg}, \mathrm{Ca} / \mathrm{K}, \mathrm{Mg} / \mathrm{K}$ y $\mathrm{Ca}+\mathrm{Mg} / \mathrm{K}$ se encuentran en equilibrio (Bertsch 1987).

La preparación del suelo fue mecánica, utilizando una arada, dos rastreadas y surcando cada $0,5 \mathrm{~cm}$.

La siembra se realizó el 24 de setiembre de 1996 utilizando semilla sexual de una colección de leguminosas forrajeras para clima frío (Cuadro 2). Se hizo corte de uniformización al inicio de las lluvias del año siguiente y se hicieron evaluaciones de producción de biomasa, altura, cobertura e incidencia de plagas y enfermedades cada seis semanas durante dos años.

La metodología de evaluación que se utilizó fue una modificación de la utilizada por la Red Internacional de Evaluación de Pastos Tropicales (RIEPT).

\section{Descripción de la unidad experimental}

La parcela experimental fue de $6 \mathrm{~m}^{2}$ con tres hileras de 3,0 $\mathrm{m}$ de largo, con una separación entre ellas de 0,5 $\mathrm{m}$. Los muestreos se hicieron en la hilera central, dejando los otros surcos como efecto de borde.

\section{Fertilización}

Las dosis aplicadas fueron 50, 50, 20, 20 $\mathrm{kg} / \mathrm{ha}$ de $\mathrm{P}_{2} \mathrm{O}_{5}, \mathrm{~K}_{2} \mathrm{O}$, Mg y S. El Fósforo, Magnesio y Azufre se incorporaron al suelo al momento de la siembra, el Potasio se aplicó

Cuadro 1. Resultado de análisis de suelo antes de la siembra. Heredia, Costa Rica. 1996.*

\begin{tabular}{|c|c|c|c|c|c|c|c|c|c|}
\hline \multicolumn{4}{|c|}{ cmol (+)/I } & \multicolumn{5}{|c|}{$\mathrm{mg} / \mathrm{l}$} & \multirow{2}{*}{$\begin{array}{c}\% \\
\text { Acidez }\end{array}$} \\
\hline pH & K & $\mathrm{Ca}$ & Mg & $\mathbf{P}$ & $\mathrm{Fe}$ & $\mathrm{Cu}$ & $\mathrm{Zn}$ & Mn & \\
\hline 5,7 & 0,4 & 4,5 & 1,5 & 11 & 193 & 6,0 & 6,1 & 10 & 3,0 \\
\hline
\end{tabular}

* Análisis de suelo realizado por el Laboratorio de Suelos, Foliares y Aguas del INTA. 
Cuadro 2. Cultivares de leguminosas que se evaluaron. Heredia, Costa Rica. 1996.

\begin{tabular}{lcll}
\hline \multicolumn{1}{c}{ Cultivares } & Simbología & Comportamiento & Procedencia \\
\hline Vicia purpurea, Púrpura & VP & Anual & Chile \\
Vicia villosa, Woolly & VV & Anual & Australia \\
Lotus corniculatus, Dewey & LD & Perenne & E.E.U.U. \\
Lotus corniculatus, Makú & LM & Perenne & Australia \\
Trifolium pratense, Renegade & TR & Semiperenne & E.E.U.U. \\
Trifolium alexandrinum, Berseen & TB & Anual & E.E.U.U. \\
Trifolium alexandrinum, Nico & TN & Anual & Chile \\
Trifolium repens, Arán & TA & Perenne & Inglaterra \\
Trifolium repens, Italia & TI & Perenne & Chile \\
Trifolium repens, Huia & TH & Perenne & Chile \\
Trifolium pratense, Safari & TS & Semiperenne & Australia \\
Trifolium pratense, Quiñequeli & TQ & Semiperenne & Chile \\
Trifolium subterraneum, Clare & TC & Perenne & Chile \\
Trifolium subterraneum, Mount Barquer & TM & Perenne & Chile \\
Trifolium repens (Testigo) & TL & Perenne & Costa Rica \\
\hline
\end{tabular}

fraccionado, con $1 / 3$ de lo recomendado cuatro semanas después de la siembra, 1/3 después del corte de uniformización y el último tercio al cabo de dos cortes (Toledo y Schultze-Kraft 1982).

\section{Variables a evaluar}

\section{Producción de biomasa y calidad nutritiva}

Como se mencionó anteriormente las evaluaciones se realizaron cada seis semanas después del corte de uniformización, la altura de corte varió de acuerdo a la morfología de las plantas, de modo que en las de hábito rastrero los cortes se hicieron a $5 \mathrm{~cm}$ sobre el nivel del suelo y las de porte alto a los $10 \mathrm{~cm}$. Los cortes se efectuaron a razón de un metro lineal en la hilera central de cada parcela, dejando sin cortar los extremos, como efecto de borde (Roig 1989).

El material de la hilera central se pesó en verde y luego una sub-muestra de $500 \mathrm{~g}$ se utilizó para la determinación del contenido de la materia seca (M.S.). Por falta de presupuesto, se utilizó una muestra compuesta de las tres repeticiones para la determinación de proteína cruda (P.C.) y digestibilidad in vitro de la materia seca (D.I.V.M.S.). Debido a esta situación se hizo análisis de varianza solamente para la producción de biomasa.

\section{Cobertura}

Esta variable se evaluó como porcentaje del área que no presenta suelo desnudo. Las evaluaciones coincidieron con los muestreos de rendimiento. Para este propósito, se utilizó la metodología propuesta por la RIEPT (Toledo y Schultze-Kraft 1982) con la diferencia de que al tener las parcelas solo tres hileras, de las cuales una constituye la parcela útil, se utilizó un marco rectangular de $0,5 \mathrm{~m}$ * $1,0 \mathrm{~m}$, con cuadrículas de $0,25 \mathrm{~m}$ * $0,25 \mathrm{~m}$.

\section{Altura}

La variable altura se midió con la misma frecuencia que la cobertura. Para esta medición, se tomó la altura desde el nivel del suelo hasta el punto más alto de la planta, sin estirarla y sin considerar la inflorescencia (Toledo, Schultze-Kraft 1982).

Plagas (insectos y enfermedades) (Adaptado de Calderón 1982 y Lenne 1982)

Se utilizó una escala de uno a cuatro en la cual:

$1=$ Presencia ( $5 \%$ del follaje afectado)

2 = Daño leve (5-20\% afectado)

3 = Daño moderado (20-40\% afectado)

$4=$ Daño grave (40\% afectado) 


\section{RESULTADOS Y DISCUSIÓN}

\section{Producción de biomasa y valor nutritivo}

Los cultivares anuales fueron cosechados al inicio de la floración (176 días de crecimiento). La Vicia purpurea, Púrpura, fue el cultivar de mayor producción de biomasa en base seca, 6,3 t/ha valor inferior a 7,1 t/ha reportado por Mesén (en prensa) y superior a 4,3 t/ha reportado por Rodríguez (1979) para Vicia villosa, Namoy. El Trifolium alexandrinum, Nico fue el de menor producción 2,7 $\mathrm{t} /$ /ha (Cuadro 3).

El Cuadro 4 muestra la producción promedio de materia seca, cobertura, altura y contenido de proteína de las leguminosas perennes o semiperennes.
Con los resultados de producción de biomasa (Cuadro 4) se realizó un análisis de varianza el cual demostró que hubo diferencias entre cultivares y entre las diferentes evaluaciones $(P=0,004$ y $P=0,0001)$. Además fueron significativas las interacciones bloque*cultivar y cultivar*evaluación ( $\mathrm{P}=0,007$ y $\mathrm{P}=0,0008)$. La prueba de Waller-Duncan (Figura 1) mostró que el cultivar con mayor producción de materia seca fue el Trifolium pratense, Renegade, con 2,3 t/ha/corte, siendo significativamente diferente a los demás. El de menor producción $(0,9 \mathrm{t} / \mathrm{ha} / \mathrm{corte})$ fue el Trifolium repens, Arán. El testigo local (Trifolium repens) desapareció aunque fue resembrado, sin embargo en la zona se encuentra en asociación con Kikuyo (Pennisetum clandestinum) en forma natural.

Cuadro 3. Comportamiento de las leguminosas anuales. Heredia, 1996.

\begin{tabular}{lcccccc}
\hline Cultivar & $\begin{array}{c}\text { P.C., } \\
\%\end{array}$ & $\begin{array}{c}\text { D.I.V.M.S., } \\
\%\end{array}$ & $\begin{array}{c}\text { M.S., } \\
\text { t/ha }\end{array}$ & $\begin{array}{c}\text { M.S., } \\
\text { kg/ha/día }\end{array}$ & $\begin{array}{c}\text { Cobertura, } \\
\%\end{array}$ & $\begin{array}{c}\text { Altura, } \\
\text { cm }\end{array}$ \\
\hline VP & 23,1 & 68,9 & 6,3 & 37,9 & 100 & 67 \\
VV & 23,1 & 70,4 & 5,0 & 29,9 & 100 & 62 \\
TB & 13,0 & 70,5 & 3,1 & 18,8 & 85 & 65 \\
TN & 13,1 & 69,5 & 2,7 & 16,4 & 75 & 69 \\
\hline
\end{tabular}

Cuadro 4. Comportamiento de los forrajes perennes o semiperennes. Heredia, 1996.

\begin{tabular}{|c|c|c|c|c|c|c|c|c|}
\hline \multirow[t]{3}{*}{ Cultivar } & \multicolumn{8}{|c|}{ Evaluaciones } \\
\hline & \multicolumn{4}{|c|}{ Época Iluviosa } & \multicolumn{4}{|c|}{ Época seca } \\
\hline & $\begin{array}{c}\text { Proteína, } \\
\%\end{array}$ & $\begin{array}{l}\text { Altura, } \\
\text { cm }\end{array}$ & $\begin{array}{c}\text { Cobertura, } \\
\%\end{array}$ & $\begin{array}{c}\text { Materia seca, } \\
\text { t/ha/corte }\end{array}$ & $\begin{array}{c}\text { Proteína, } \\
\%\end{array}$ & $\begin{array}{c}\text { Altura, } \\
\text { cm }\end{array}$ & $\begin{array}{c}\text { Cobertura, } \\
\%\end{array}$ & $\begin{array}{c}\text { Materia seca, } \\
\text { t/ha/corte }\end{array}$ \\
\hline LD & - & - & - & $\mathrm{P}$ & - & - & - & $\mathrm{P}$ \\
\hline LM & 27,5 & 21,0 & 93,0 & 1,5 & 24,7 & 16,0 & 85,0 & 0,6 \\
\hline TR & 26,3 & 43,0 & 96,0 & 2,3 & 25,2 & 34,0 & 92,0 & 1,3 \\
\hline TA & 28,0 & 23,0 & 65,0 & 0,9 & 23,2 & 23,0 & 55,0 & 0,7 \\
\hline $\mathrm{TI}$ & 27,4 & 26,0 & 94,0 & 1,4 & 22,7 & 26,0 & 90,0 & 0,9 \\
\hline $\mathrm{TH}$ & 25,8 & 20,0 & 76,0 & 1,0 & 22,5 & - & - & $\mathrm{P}$ \\
\hline TS & 24,8 & 23,0 & 57,0 & 1,6 & - & - & - & $P$ \\
\hline TQ & 22,7 & 35,0 & 84,0 & 1,7 & 25,1 & 26,0 & 74,0 & 1,0 \\
\hline TC & - & - & - & $\mathrm{P}$ & - & - & - & $\mathrm{P}$ \\
\hline $\mathrm{TM}$ & - & - & - & $\mathrm{P}$ & - & - & - & $\mathrm{P}$ \\
\hline Testigo & 25,5 & 18,0 & 60,0 & 0,6 & 25,5 & - & - & $\mathrm{P}$ \\
\hline
\end{tabular}

$\mathrm{P}=$ perdido. 


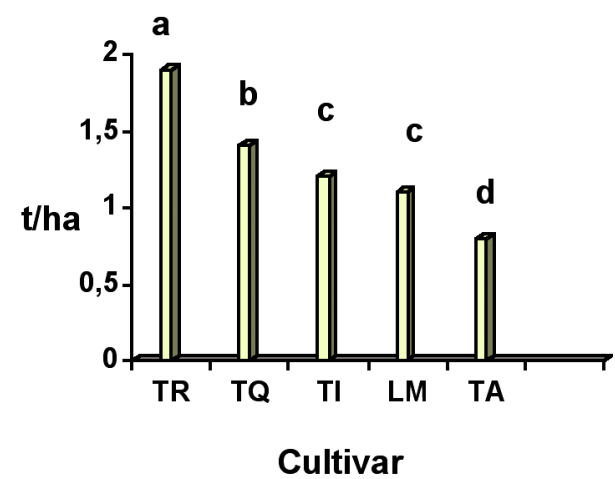

Letras iguales no difieren entre sí $(P \geq 0,05)$

Figura 1. Producción de biomasa en base seca. Heredia, Costa Rica. 1996.

Letras iguales no difieren entre sí $(P \geq 0,05)$.

El Cuadro 5 muestra los valores promedio de la proteína cruda, los cuales fueron similares en los diferentes cultivares, oscilando en un rango de 23,9 a 26,1 \%. Además presentan valores similares en las dos épocas del año.

Cuadro 5. Contenido porcentual de proteína cruda según época de muestreo. Heredia, 1996.

\begin{tabular}{cccc}
\hline Cultivar & Época lluviosa & Época seca & Promedio \\
\hline LD & 27,3 & 24,2 & 25,7 \\
LM & 27,5 & 24,7 & 26,1 \\
TR & 26,3 & 25,2 & 25,7 \\
TA & 28,0 & 23,2 & 25,6 \\
TI & 27,4 & 22,7 & 25,0 \\
TH & 25,8 & 22,5 & 24,1 \\
TS & & $\mathrm{P}$ & \\
TQ & 22,7 & 25,1 & 23,9 \\
TC & $\mathrm{P}$ & & \\
TM & $\mathrm{P}$ & & \\
Testigo & 25,5 & 25,5 & 25,5 \\
\hline
\end{tabular}

$\mathrm{P}=$ perdido

\section{Cobertura}

Los cultivares anuales presentaron valores de cobertura superiores al $75 \%$ (Cuadro 3). Los cultivares perennes o semiperennes que no se perdieron presentaron valores variados de cobertura, el cultivar de mayor cobertura fue el Trifolium pratense, Renegade con un $95 \%$ de suelo cubierto, el de menor cobertura fue el Trifolium repens, Arán con un $50 \%$ (Cuadro 6). Estos datos están correlacionados con la producción de biomasa.

Cuadro 6. Promedios de altura y cobertura de los cultivares persistentes. Heredia, 1996.

\begin{tabular}{ccc}
\hline Cultivar & Altura, cm & Cobertura, \% \\
\hline LM & 19 & 85 \\
TR & 40 & 95 \\
TA & 18 & 50 \\
TI & 25 & 90 \\
TQ & 30 & 85 \\
\hline
\end{tabular}

\section{Altura}

Los valores de altura en los cultivares anuales fueron muy similares, oscilaron en un rango de 62 a $69 \mathrm{~cm}$. Los demás cultivares persistentes presentaron valores variados, el de mayor altura fue el Trifolium pratense, Renegade con $40 \mathrm{~cm}$ y el más bajo Trifolium repens, Arán con $18 \mathrm{~cm}$ (Cuadro 6).

\section{Plagas (insectos y enfermedades)}

Ninguno de los cultivares presentó daños causados por insectos o por enfermedades.

\section{CONCLUSIONES Y RECOMENDACIONES}

Teniendo en consideración las condiciones bajo las cuales se llevó a cabo el ensayo, se pueden formular las siguientes conclusiones y recomendaciones:

- Los cultivares anuales dieron buenos resultados, las altas tasas de crecimiento por hectárea, demuestran la adaptabilidad de los mismos a la zona. 
- De los cultivares perennes o semiperennes que se evaluaron, sobrevivieron únicamente cinco, siendo el de mayor producción el Trifolium pratense, Renegade, y el de menor producción el Trifolium repens, Arán.

- De los cultivares semiperennes, sobrevivieron los Trifolium pratenses, Renegade y Quiñequeli.

- De los cultivares perennes el Trifolium repens, Italia y el Lotus corniculatus, Makú, fueron los de mayor producción de materia seca, por lo cual se deben estudiar en asociación con gramíneas y bajo pastoreo

- Los demás cultivares evaluados no se adaptan como monocultivo bajo corte.

- Se debe continuar investigando con leguminosas de mayor potencial productivo.

\section{LITERATURA CITADA}

Bernal, J. 1991. Pastos y forrajes tropicales $2^{\circ}$ Edición. Colombia. Banco Ganadero. p. 273.

Bertsch, F. et al. 1993. Características de los principales órdenes de suelos presentes en Costa Rica. Congreso Nacional Agropecuario y de Recursos Naturales. UCR.Costa Rica. 78 p.

Bertsch, F. 1987. Manual para interpretar la fertilidad de los suelos de Costa Rica. Universidad de Costa Rica. Costa Rica. 82 p.

Calderón, M. 1982. Evaluación del daño causado por insectos. In: Toledo, J.M. ed. Manual para la evaluación agronómica. Red Internacional de Evaluación de Pastos Tropicales. (RIEPT), CIAT, Cali, Colombia. p. 45-56.
Lenne, J. 1982. Evaluación de enfermedades en pastos tropicales. In: Toledo, J.M. Manual para la evaluación agronómica. Red Internacional de Evaluación de Pastos Tropicales. (RIEPT), CIAT, Cali, Colombia. p. 57-72.

MAG (Ministerio de Agricultura y Ganadería, CR); IICA (Instituto Interamericano de Cooperación para la Agricultura, CR). 1991. Estudio a nivel nacional para mejorar la tecnología de alimentación del ganado lechero. Costa Rica. p. 265.

Mesén, M. Evaluación de leguminosas herbáceas de uso potencial en sistemas de producción de leche de altura. En prensa.

Roig, C.A. 1989. Evaluación preliminar de 200 accesiones de leguminosas forrajeras tropicales en el ecosistema de Bosque Tropical Lluvioso en Costa Rica. Guápiles, Costa Rica. Tesis Mag. Sc. CATIE, Turrialba, Costa Rica. 179 p.

Rodríguez, H. 1979. Determinación de la densidad de siembra de la Veza vellosa como abono verde y su efecto en la producción de sorgo de grano. Tesis Ing. Agr. Universidad Autónoma de Nuevo León. Facultad de Agronomía. México. $31 \mathrm{p}$.

Toledo, J.M.; Schultze - Kraft, R. 1982. Metodología para la evaluación agronómica de pastos tropicales. In: Toledo, J.M. ed. Manual para la Evaluación Agronómica. Red Internacional de Evaluación de Pastos Tropicales (RIEPT), CIAT, Cali, Colombia. p. 91-109.

Villegas, L. 2004. Factores determinantes de la productividad de fincas lecheras en pastoreo In: Memoria del Seminario de ganadería de leche. INTA. Departamento de Transferencia. Costa Rica. sp. 